\title{
Die Verantwortung wissenschaftlicher Bibliotheken im Zeitalter der Digitalisierung
}

\begin{abstract}
Die Digitalisierung in allen Lebensbereichen birgt neben bis vor Kurzem ungeahnten neuen Möglichkeiten ebenso völlig neue Herausforderungen. Nicht nur in der Wirtschaft (Stichwort „Industrie 4.0“), sondern sogar mehr noch für Wissenschaftsstandorte wird die Fähigkeit zu einem guten Umgang mit der Digitalisierung zunehmend zu einer entscheidenden Voraussetzung für künftige Wettbewerbsfähigkeit. Eine wesentliche Rolle spielt dabei die breite Nutzung von Forschungsdaten weit über deren ursprünglichen Erhebungszweck hinaus. Aus dieser Perspektive werden die zentralen Handlungsfelder Dienstleistungen für und durch das Forschungsdatenmanagement, Datenqualität, digitale Langzeitarchivierung sowie Lehre, Informationskompetenz und Nachwuchsförderung beleuchtet. Auf dieser Grundlage wird herausgearbeitet, dass diese Entwicklungen für die wissenschaftlichen Bibliotheken bedeuten, dass sie nicht nur auf den Feldern ihrer traditionellen Aufgaben gefordert sind, sich diesen Herausforderungen zu stellen, sondern dass sie besonders gefordert sind, bei der Gestaltung der neuen Herausforderungen für die Wissenschaft insgesamt und insbesondere im Forschungsdatenmanagement eine aktiv gestaltende Rolle zu übernehmen. Darauf gestützt wird die These formuliert, dass hier aktuell ein Aufgabenbereich entsteht, in welchem wissenschaftliche Bibliotheken mit ihren traditionellen Kernkompetenzen in der systematischen ErschlieBung und dauerhaften Verfügbarmachung publizierter Informationen besondere Verantwortung tragen und dass dieser Bereich sich schon in wenigen Jahren als für das wissenschaftliche Bibliothekswesen profilprägend erweisen wird. Dabei ist eine enge Verzahnung von Fachwissenschaften, von Informatik und Rechenzentren sowie der wissenschaftlichen Bibliotheken notwendig.
\end{abstract}

\section{Digitalisierung als Zeitalter großer Herausforderungen}

Herausforderungen durch die neuen digitalen Möglichkeiten: Zunehmende Digitalisierung verändert nicht nur unseren Alltag und das Wirtschaftsleben grundlegend, sondern in noch einmal gesteigerten Maße die Wissenschaft. Allein die 
Hälfte des Wissens, mit dem wir heute arbeiten, wurde in den letzten beiden Jahren erzeugt. ${ }^{1}$ Diese Informationsflut ist jedoch kein reines Mengen- oder Volumenproblem, wie der modische, aber missverständliche Begriff Big Data glauben machen könnte. Sicher brauchen größere Datenmengen auch größere Speicher-, Rechen- und Leitungskapazitäten. Viel entscheidender aber ist, dass Digitalisierung nicht etwa nur ein neues Werkzeug zur Bearbeitung einer ansonsten unveränderten Forschungsagenda ist, sondern dass die Digitalisierung die Forschung selber von Grund auf transformiert. ${ }^{2}$ Erst durch die Digitalisierung entstanden praktikable Möglichkeiten, auf Daten unterschiedlichster Herkunft effektiv zugreifen zu können und diese wirkungsvoll untereinander zu verknüpfen. Damit können völlig neue Formen von Forschung realisiert werden und neue Forschungsgegenstände erschließen sich für eine Bearbeitung. So ist dank der Simulationswissenschaft ein realitätsnaher Test von Neukonstruktionen nicht mehr erst mit dem physischen Bau eines Demonstrators möglich, sondern dieser kann bereits viel früher und ressourcensparend auf dem Rechner simuliert werden. In der Klima- und Umweltforschung wird eine gesamthafte Betrachtung systemischer Zusammenhänge erst durch die Verknüpfung unterschiedlicher Datenquellen möglich. In der Medizin gilt Entsprechendes für das hochkomplexe, von vielen Wirkungsfaktoren beeinflusste System Mensch; erst so wird ein personalisierter Ansatz möglich, der die individuellen Merkmale des oder der zu Behandelnden, deren sozialen Kontext und weitere Informationen immer besser mit dem ärztlichen Erfahrungswissen verbindet. ${ }^{3}$ Voraussetzungen für eine ressourcenschonende, ökologische und zugleich ökonomisch tragfähige Landwirtschaft werden durch intelligente Verknüpfung von Daten geschaffen. Zugleich stellen sich nicht nur für Juristen und Philosophen völlig neue Fragen, wie Rahmenbedingungen und Verantwortungssysteme so gestaltet werden können, dass solche Chancen genutzt und gleichzeitig mögliche Fehlentwicklungen wirksam vermieden werden. Weiterhin entwickelt sich innerwissenschaftlich das Verständnis von guter wissenschaftlicher Praxis fort.

1 Vgl. Bürger, Thomas: Die Explosion des Wissens. Wie Informationsflut und Technikbeschleunigung Bibliotheken verändern. In: BIS - Das Magazin der Bibliotheken in Sachsen 8 (2015) H. 1. S. 44-46.

2 In diesem Sinne auch aktuell das Positionspapier der Deutschen Forschungsgemeinschaft: Förderung von Informationsinfrastrukturen für die Wissenschaft. Bonn, 15. März 2018, S. 12. http://www.dfg.de/download/pdf/foerderung/programme/lis/positionspapier_informationsinfrastrukturen.pdf (6.4.2018).

3 Dazu ausführlich der Zukunftsreport Lebenswissenschaften im Umbruch der Leopoldina von 2014: https://www.leopoldina.org/uploads/tx_leopublication/2014_Zukunftsreport_Langfassung_web.pdf sowie die aktuellen Aktivitäten im Rahmen der von der Bundesregierung geförderten Medizininformatik-Initiative. 
Die längerfristige Aufbewahrung von Publikationen zugrunde liegenden Forschungsdaten gehört inzwischen zum Kanon guter wissenschaftlicher Praxis; ${ }^{4}$ die mangelnde Reproduzierbarkeit von Versuchsreihen und die mangelnde Verfügbarkeit von Forschungsdaten, die zu einem negativen, die zugrunde liegende Forschungshypothese widerlegenden, Ergebnis geführt haben, werden hingegen vielfach beklagt.

Grundlegend gewandeltes wissenschaftliches Informations- und Publikationsverhalten verlangt neue Angebote: Zudem ist zu berücksichtigen, dass sich in den letzten Jahren das Informations- und Publikationsverhalten in allen Bereichen grundlegend verändert hat. Während noch bis vor Kurzem die Konsumption aufbereiteter Informationskorpora wie gedruckte Bücher, Zeitungen und Zeitschriften sowie wenige Radio- und Fernsehsender mit festen Programmschemata im Vordergrund stand, herrscht heutzutage eine nahezu unüberschaubare Fülle an Informationen aus unterschiedlichsten Quellen vor, die es zu bewerten und einzuordnen gilt. Zudem sind die Möglichkeiten, nicht aufbereitete Informationen verfügbar $\mathrm{zu}$ machen, durch das Internet exponentiell gewachsen ebenso wie die Nachfrage, solche Informationen bereits im unbearbeiteten Originalzustand und in Echtzeit nutzen zu können.

Ebenso verändert sich das Publikationsverhalten fundamental, indem $\mathrm{Pu}$ blikationen nicht mehr erst mit ihrem Erscheinen in traditionellen Organen öffentlich werden und Daten längst nicht mehr nur als unselbständige Anhänge zu Publikationen erscheinen, sondern immer systematischer in einschlägigen Repositorien abgelegt werden. Auch dies entspricht der Bedarfsentwicklung der Nutzerschaft. Nicht zuletzt hat die weit überzogene Ausnutzung der Quasimonopolstellung einzelner Großverlage in wichtigen Segmenten diese Entwicklung weiter beschleunigt, indem sie zu einem deutlichen Akzeptanzverlust traditioneller Publikationsformen nicht nur bei jüngeren Wissenschaftlern geführt hat. Überdies werden klassische, Wissenschaftskarrieren noch heute entscheidend prägende Reputationsmechanismen wie schlichte Zitationsindizes aus marktstarken, aber stark selektiven und damit leistungsverzerrenden e-Datenbanken zunehmend infrage gestellt. ${ }^{5}$

\footnotetext{
4 Vgl. Deutsche Forschungsgemeinschaft: Sicherung guter wissenschaftlicher Praxis. Denkschrift. Empfehlungen der Kommission „Selbstkontrolle in der Wissenschaft“. Bonn 2013. http://www.dfg.de/download/pdf/dfg_im_profil/reden_stellungnahmen/download/empfehlung_wiss_praxis_1310.pdf (21.12.2017).

5 Vgl. zuletzt die gemeinsame Stellungnahme „on good practice in the evaluation of researchers and research programmes“ der britischen Royal Academy, der französischen Académie des sciences sowie der Leopoldina, 27. Oktober 2017. http://www.leopoldina.org/uploads/ tx_leopublication/2017_Statement_3Acad_Evaluation.pdf (21.12.2017).
} 
Adäquate wissenschaftliche Reputationsmechanismen sind noch zu schaffen: Allerdings tragen die innerwissenschaftlichen Reputationsmechanismen, die weiterhin sehr umfangreich auf klassischen Publikationen aufsetzen, diesen Veränderungen noch kaum Rechnung. Die aktuelle Bibliometrie verliert damit als Indikator wissenschaftlicher Leistungen zunehmend an Wert und rein publikations- und zitationsbasierte Indikatoren werden mit weiterem Fortgang der Entwicklung zu einem Hemmnis für wissenschaftlichen Fortschritt, sofern es nicht gelingt, die neuen technischen Möglichkeiten auch in der Bibliometrie dafür zu nutzen, neue qualitätsbasierte Indikatoren zu entwickeln, die wissenschaftliche Leistung der veränderten wissenschaftlichen Praxis besser entsprechend zu beschreiben und einen Informed Peer Review wirkungsvoll zu unterstützen vermögen. ${ }^{6}$

Auch die Aufgaben wissenschaftlicher Bibliotheken befinden sich im Wandel: Für die wissenschaftlichen Bibliotheken bedeuten die geschilderten Entwicklungen, dass sich Bibliotheksleistungen - wie sie traditionell vorrangig über effektiver Arbeit förderliche Leseräume, reichhaltige Printbestände in gut zugänglichen Freihandbereichen und geschützten Magazinen, erschlossen durch alphabetische und systematische Kataloge sowie eine kompetente Fachauskunft in beeindruckender Breite und Qualität geboten wurden und werden und die in der Folge durch effektive Systeme der Fernleihe, Systeme schneller Aufsatzlieferungen sowie Bezug und Vermittlung elektronischer Medien ergänzt wurden - diesen Veränderungen anpassen und Bibliotheken ihr Missionsverständnis in das digitale Zeitalter übersetzen müssen. Dabei liegt der Kern dieser Aufgabe nicht darin, digitale Werkzeuge zur weiteren Erfüllung ihrer klassischen Aufgaben einzusetzen und klassische Werkzeuge auf digitale Publikationen anzuwenden. Vielmehr besteht die zentrale Frage darin, welche neuen Aufgaben für Bibliotheken aus dieser Entwicklung entstehen und welche Beiträge sie zur Gestaltung und Beherrschung der Veränderungen leisten. Wie im Folgenden zu zeigen sein wird, liegt ein zentrales Handlungsfeld, das schon in wenigen Jahren das Profil wissenschaftlicher Bibliotheken prägen wird, im Forschungsdatenmanagement.

6 Vgl. dazu Arbeitsgemeinschaft der Wissenschaftlichen Medizinischen Fachgesellschaften: Positionspapier zur Evaluierung der medizinischen Forschungsleistung, 26. Juni 2014. http:// www.egms.de/static/de/journals/gms/2014-12/000196.shtml sowie die Stellungnahme der drei Wissenschaftsakademien (wie Anm. 5). 


\section{Strategische Impulse für die Datenkultur konvergieren}

Die Dringlichkeit des Handlungsbedarfes im Bereich des Forschungsdatenmanagements wurde international, aber auch in Deutschland bereits frühzeitig angemahnt. Inzwischen liegt dazu national und international eine ganze Reihe strategischer Papiere vor. Einen wesentlichen Impuls lieferte ein im Jahr 2007 erschienener OECD-Bericht. ${ }^{7} 2008$ wurde in Australien der National Data Service als zentrale Dienstleistungsinstanz gegründet. Auf globaler Ebene bildete sich 2013 die Research Data Alliance (RDA). Ziel von RDA ist die Beschleunigung der internationalen datengetriebenen Innovation durch bessere Zugänglichkeit und Verknüpfbarkeit von Daten. In dieser bottom-up organisierten Bewegung arbeiten mittlerweile 6400 Fachleute aus Wissenschaft und Wirtschaft in 133 Ländern mit (Stand Januar 2018). Die erste Plenartagung in Deutschland fand im März 2018 in Berlin statt. In Europa gehörten die Technischen Universitäten zu den Schrittmachern. ${ }^{8}$ Auf europäischer Ebene machte im Jahr 2015 EU-Wissenschaftskommissar Carlos Moedas die Schaffung einer European Open Science Cloud (EOSC) bis 2020 zu einem zentralen Projekt seiner Amtszeit. Basierend auf Empfehlungen von High Level Expert Groups aus den Jahren $2010^{9}$ und $2016^{10}$ hat die Kommission inzwischen ihre Erwartungen an das Forschungsdatenmanagement in Europa in einer EOSC Declaration zusammengefasst. ${ }^{11}$ Bislang haben rund 70 Wissenschaftseinrichtungen ihre Unterstützung schriftlich zugesagt. ${ }^{12}$ Zur weiteren Umsetzung wurde von der EU-

7 The Organisation for Economic Co-operation and Development: OECD Principles and Guidelines for Access to Research Data from Public Funding. Paris 2007. https://www.oecd.org/sti/ sci-tech/38500813.pdf (27.12.2018).

8 Vgl. League of European Research Universities - Research Data Working Group: LERU Roadmap for Research Data. Advice Paper No. 14, Dezember 2013. https://www.leru.org/files/LERURoadmap-for-Research-Data-Full-paper.pdf (27.12.2018).

9 Riding the Wave. How Europe can gain from the rising tide of scientific data. Final report of the High Level Expert Group on Scientific Data. A submission to the European Commission, Oktober 2010. http://ec.europa.eu/information_society/newsroom/cf/itemlongdetail.cfm?item_id=6204 (17.12.2017).

10 Realising the European Open Science Cloud. First report and recommendations of the Commission High Level Expert Group on the European Open Science Cloud, 2016. https://ec. europa.eu/research/openscience/pdf/realising_the_european_open_science_cloud_2016.pdf (21.12.2017).

11 EOSC Declaration. Brüssel, 26. Oktober 2017. https://ec.europa.eu/research/openscience/ pdf/eosc_declaration.pdf (21.12.2017).

$12 \mathrm{https} / /$ ec.europa.eu/research/openscience/pdf/list_of_institutions_endorsing_the_eosc_declaration.pdf (21.12.2017). 
Kommission Anfang 2018 ein Arbeitsdokument vorgelegt. Auf Ebene der Mitgliedsstaaten (derzeit Deutschland, Niederlande und Frankreich) sowie europäischer Forschungsinitiativen und Netzwerke begleitet die so genannte GO-FAIRInitiative diese Vorhaben. ${ }^{13}$ In ihrem Arbeitsprogramm werden erhebliche Mittel auf die dort verfolgten Ziele konzentriert.

In Deutschland wurde die Problematik erstmalig in den Empfehlungen der Kommission für Informationsinfrastrukturen von $2011^{14}$ umfassend aufgearbeitet. Auch der Wissenschaftsrat hat die Herausforderungen frühzeitig und kontinuierlich in seinen Empfehlungen aufgegriffen. ${ }^{15}$ Die Hochschulrektorenkonferenz (HRK) folgte im Jahr 2015 mit einer ausführlichen Stellungnahme. ${ }^{16} \mathrm{Im}$ selben Jahr veröffentlichte auch die Initiative der Allianz der Wissenschaftsorganisationen ein Positionspapier. ${ }^{17}$ Wissenschaftspolitische Dynamik erhielt das Thema allerdings aufgrund der Arbeiten des im Jahre 2014 gegründeten und die Bandbreite des deutschen Wissenschaftssystems abbildenden Rates für Informationsinfrastrukturen (RfII). Ihm gehören neben je zwei Vertretern von

13 Vgl. Deutschland und die Niederlande für rasches Handeln bei der European Open Science Cloud. Pressemitteilung des Bundesministeriums für Bildung und Forschung vom 31. Mai 2017. https://www.bmbf.de/de/deutschland-und-die-niederlande-fuer-rasches-handeln-bei-der-european-open-science-cloud-4270.html (21.12.2017).

14 Kommission Zukunft der Informationsinfrastruktur: Gesamtkonzept für die Informationsinfrastruktur in Deutschland, April 2011. https://www.leibniz-gemeinschaft.de/fileadmin/user_upload/downloads/Infrastruktur/KII_Gesamtkonzept.pdf (17.12.2017).

15 Vgl. Wissenschaftsrat: Empfehlungen zur Weiterentwicklung der wissenschaftlichen Informationsinfrastrukturen in Deutschland bis 2020. Berlin, 13. Juli 2012. http://www.wissenschaftsrat.de/download/archiv/2359-12.pdf (21.12.2017); Wissenschaftsrat: Bericht zur wissenschaftsgeleiteten Bewertung umfangreicher Forschungsinfrastrukturvorhaben für die Nationale Roadmap (Pilotphase). Berlin 2013. http://www.wissenschaftsrat.de/download/archiv/ 2841-13.pdf (21.12.2017); Wissenschaftsrat: Bedeutung und Weiterentwicklung von Simulation in der Wissenschaft. Positionspapier. Dresden 2014. https://www.wissenschaftsrat.de/download/archiv/4032-14.pdf (21.12.2017); Wissenschaftsrat: Empfehlungen zur Finanzierung des Nationalen Hoch- und Höchstleistungsrechnens in Deutschland. Stuttgart, 24. April 2015. http:// www.wissenschaftsrat.de/download/archiv/4488-15.pdf (21.12.2017); Wissenschaftsrat: Empfehlungen zur Spezifikation des Kerndatensatz Forschung. Berlin, 22. Januar 2016. http:// www.wissenschaftsrat.de/download/archiv/5066-16.pdf (21.12.2017).

16 Hochschulrektorenkonferenz: Empfehlung der 19. Mitgliederversammlung der HRK am 10. November 2015 in Kiel. Wie Hochschulleitungen die Entwicklung des Forschungsdatenmanagements steuern können. Orientierungspfade, Handlungsoptionen, Szenarien. Bonn 2015. https://www.hrk.de/fileadmin/_migrated/content_uploads/Empfehlung_Forschungsdatenmanagement_final_Stand_11.11.2015.pdf (15.9.2017).

17 Schwerpunktinitiative „Digitale Information“ der Allianz der deutschen Wissenschaftsorganisationen, Positionspapier „Research data at your fingertips“ der Arbeitsgruppe Forschungsdaten, Februar 2015. http://gfzpublic.gfz-potsdam.de/pubman/item/escidoc:986897:6/component/escidoc:986898/Positionspapier_Forschungsdaten.pdf (17.12.2017). 
Bund und Ländern sowie vier Vertretern des öffentlichen Lebens ausschließlich Wissenschaftler an, die sich aus der disziplinären und institutionellen Vielfalt des deutschen Wissenschaftssystems rekrutieren und sich je zur Hälfte aus Vertretern der Infrastrukturanbieter sowie der Infrastrukturnutzer zusammensetzen. Aus den Reihen der wissenschaftlichen Bibliotheken gehörte Thomas Bürger zu den Gründungsmitgliedern. Im Jahr 2016 legte der RfII seine grundlegenden Empfehlungen Leistung aus Vielfalt vor. ${ }^{18}$ Zentraler Gegenstand der Empfehlungen ist der Aufbau einer nachhaltig finanzierten Forschungsinfrastruktur. Diese soll vorhandene Strukturen und Initiativen aber nicht doppeln, sondern aufbauend auf dem Vorhandenen deren Zusammenwachsen zu einem über Grenzen von Disziplinen und Institutionen synergetisch interagierenden Netzwerk fördern. Dieses Zusammenwachsen wird als ein langfristiger Prozess mit einem Zeithorizont von 20 bis 25 Jahren angesehen. Eine entsprechende Ausschreibung der Gemeinsamen Wissenschaftskonferenz wird für das Jahr 2018 erwartet.

Impulse für die Arbeit wissenschaftlicher Bibliotheken: Forschungsdatenmanagement zählt nicht zum hergebrachten Markenkern von Bibliotheken, wohl aber die Sicherung der Qualität der Informationsversorgung. ${ }^{19}$ Bibliotheken waren von Anbeginn der Erfindung der Schrift Pioniere in der Ordnung und Erschließung heterogener Informationsbestände, für deren sichere und dauerhafte Aufbewahrung sowie für die Schaffung guter Umgebungen für wissenschaftliches Arbeiten. Alle diese Kernkompetenzen werden auch für das Forschungsdatenmanagement gebraucht. So ist es bezeichnend, dass besonders in Nordamerika (Cyberinfrastructure Initiative in Kanada und NIH Commons in den Vereinigten Staaten) und in den Niederlanden wissenschaftliche Bibliotheken eine führende Rolle bei der Gestaltung der mit dem enormen Anwachsen von Datenbeständen einhergehenden Herausforderungen übernahmen..$^{20}$ Aktuell macht auch die europäische Bibliothekenvereinigung LIBER das Forschungsdatenmanagement $\mathrm{zu}$

18 Rat für Informationsinfrastrukturen: Leistung aus Vielfalt. Empfehlungen zu Strukturen, Prozessen und Finanzierung des Forschungsdatenmanagements in Deutschland. Göttingen 2016. http://www.rfii.de/de/category/dokumente/ (17.12.2017).

19 Vgl. Martin, Christiane Laura: Wissenschaftliche Bibliotheken als Akteure im Forschungsdatenmanagement. In: LIBREAS. Library Ideas H. 2/2013 (Nr. 23). S. 12-21. libreas.eu/ausgabe23/03martin (21.12.2017).

20 Vgl. Rat für Informationsinfrastrukturen: Entwicklung von Forschungsdateninfrastrukturen im internationalen Vergleich. Bericht und Anregungen. Göttingen 2017. http://www.rfii.de/de/ category/dokumente/ (17.12.2017). 
einem zentralen Thema ihrer Strategie. ${ }^{21}$ In Deutschland sprach die Allianz der deutschen Wissenschaftsorganisationen in ihrem bereits erwähnten Positionspapier von 2015 die wissenschaftlichen Bibliotheken direkt an, ohne ihnen allerdings eine exklusive Rolle einzuräumen:

Vergleichbar mit Bibliotheken und ihrer Informationskompetenz bei der Literaturversorgung bedarf es für die nachhaltige Versorgung der Wissenschaft mit Forschungsdaten der Etablierung von vertrauenswürdigen (trustworthy) rechtsverbindlichen Organisationsstrukturen. Ideal wäre der Auf- und Ausbau von fachwissenschaftlichen Forschungsdatenzentren. 22

Es handelt sich also um Aufgaben, für die Bibliotheken mit ihren klassischen Kernkompetenzen geradezu prädestiniert sind, welche aber ein Zusammenwirken verschiedener Akteure erfordert und welche angesichts ihrer Dringlichkeit notfalls sogar parallel zu vorhandenen bibliothekarischen Strukturen organisiert werden müssen. Ähnlich sieht der Rat für Informationsinfrastrukturen in seinen Empfehlungen Leistung aus Vielfalt hier eine wichtige, aber nicht ausschließliche Rolle wissenschaftlicher Bibliotheken. ${ }^{23}$ Trotz sehr überzeugender Beispiele aus den eigenen Reihen - auch die von Thomas Bürger geleitete Sächsische Landesbibliothek - Staats- und Universitätsbibliothek Dresden (SLUB) gehört hier zu den Vorreitern ${ }^{24}$ - benötigten die wissenschaftlichen Bibliotheken als Gesamtheit hingegen erstaunlicherweise deutlich länger, um sich ebenso nachdrücklich zu ihrer Verantwortung im Forschungsdatenmanagement $\mathrm{zu}$ bekennen; im Herbst 2017 hebt jedoch die Sektion 4 des Deutschen Bibliotheksverbandes (Wissenschaftliche Bibliotheken) in ihrem Strategiepapier Wissenschaftliche Bibliotheken 2025 das Forschungsdatenmanagement als eine zentrale Zukunftsaufgabe hervor. ${ }^{25}$

In den folgenden Abschnitten sollen nun einige der wesentlichen Aspekte dieses Handlungsfeldes näher beleuchtet werden.

21 LIBER Europe: Research Libraries Powering Sustainable Knowledge in the Digital Age. Den Haag, 16. November 2017. http://libereurope.eu/wp-content/uploads/2017/11/LIBER-Strategy2018-2022.pdf (17.12.2017).

22 Schwerpunktinitiative „Digitale Information“ (wie Anm. 17), S. 4.

23 Vgl. RfII, Leistung aus Vielfalt (wie Anm. 18), S. 19 u. 44.

$24 \mathrm{Vgl}$. https://slub-dresden.de/service/forschungsdaten.

25 In der Plenarversammlung im November 2017 wurde das Papier beschlossen. http://www. bibliotheksverband.de/fileadmin/user_upload/Sektionen/sektion4/Publikationen/ WB2025_Endfassung_endg.pdf (6.4.2018). 


\section{Dienstleistungen für und durch das Forschungsdatenmanagement}

Anforderungen der Nutzerschaft: Wie die Hochschulrektorenkonferenz in ihrer Empfehlung von 2015 betont, sind Wissenschaftlerinnen und Wissenschaftler heute auf eine Vielzahl von Services angewiesen, die ihnen das Management von Forschungsdaten über den gesamten Data Life Cycle hinweg erleichtern, d. h. mit Blick auf das Produzieren, Transportieren, Prozessieren, Beschreiben, Publizieren, Darstellen und (Langzeit-)Archivieren der Daten. Diese Dienstleistungen sind nach Auffassung der HRK so auszurichten, dass die Wissenschaftlerinnen und Wissenschaftler an jedem Punkt des Data Life Cycle die adäquate Unterstützung finden und auf Doppelarbeiten verzichten können. Die Services sollten dabei bestmöglich in die Arbeits- und Forschungsumgebung der Wissenschaftlerinnen und Wissenschaftler integriert werden. ${ }^{26}$

Konsequenzen für Dienstleistungsanbieter: Zusammen mit den im vorigen Abschnitt beleuchteten Herausforderungen bedeutet dies eine wachsende Verantwortung gerade der wissenschaftlichen Bibliotheken, Informationen in ihrer Vielfalt nicht nur überhaupt verfügbar zu machen, sondern - ganz in Weiterführung ihrer grundlegenden historischen Rolle - effektive Unterstützung bei der Ermittlung der für den jeweilig Nutzenden relevanten Informationen einschließlich der zur Bewertung und Einordnung erforderlichen „Metadaten“ zu bieten sowie vielfältig nutzbare Verknüpfungsmöglichkeiten $\mathrm{zu}$ schaffen, um solche Informationen mit Informationen aus ganz anderen Wissensbeständen zu neuen Gedankensystemen zusammenfügen zu können. Die Erschließung und Vermittlung von Zugängen zu Forschungsdaten werden in absehbarer Zeit wichtiger als die vergleichbaren Aufgaben im Zusammenhang mit klassischen Publikationen. Konkret bedeutet dies die Vermittlung eines möglichst vollständigen digitalen Zugangs zu einem zunehmend virtuellen Bestand, auch zu anderenorts gespeicherten Datenbeständen, neue Informationsdienstleistungen wie z. B. Discovery-Systeme auch für Forschungsdaten, Förderung der Publikation von Forschungsdaten möglichst in Open Access, eine aktiv gestaltende Rolle bei der Etablierung eines systematischen Forschungsdatenmanagements sowie nicht zuletzt die Fortentwicklung der Methodik (dazu unten Ziff. 4), die Entwicklung und Pflege relevanter Standards (dazu unten Ziff. 5), die Bewahrung auch des digitalen kulturellen Erbes und die Organisation einer effektiven digitalen Langzeitarchivierung (dazu unten Ziff. 6) sowie die Vermittlung von Informationskompetenz (dazu unten Ziff. 7) zu diesen neuen Feldern. Solche

26 Vgl. HRK, Empfehlung (wie Anm. 16), insbesondere Ziff. 2.5. 
Anpassungen an die Bedarfsentwicklung sind unumgänglich, um auf entscheidenden Handlungsfeldern auch künftig die Position eines für wissenschaftlichen Erfolg relevanten Akteurs zu behaupten.

Zugleich bietet die Verknüpfung von Daten übrigens enorme Potenziale zur Verbesserung der eigenen Services von Bibliotheken, etwa bei der Verbesserung der Zielgenauigkeit von Informationsangeboten oder bei der Erarbeitung von Qualitätsindikatoren für wissenschaftliche Leistungen über den aktuellen Kanon von Impact-Faktoren hinaus, die weder dem sich ändernden Informationsund Publikationsverhaltens gerecht werden noch die Bandbreite wissenschaftlicher Leistungen etwa in den Bereichen Infrastruktur, Transfer und Nachwuchsförderung $\mathrm{zu}$ beleuchten vermögen. Gerade im Zeitalter der Informationsflut im Internet wird ebenso die qualitätsgesicherte, evidenz- und faktenbasierte Informationsversorgung der Öffentlichkeit zu einem immer größeren Desiderat.

Daneben ist die Fortentwicklung des Angebotsportfolios auch zur Pflege der Nutzerbeziehungen unumgänglich. Die traditionell privilegierte Sichtbarkeit wissenschaftlicher Bibliotheken bei der Nutzerschaft - welcher Forschende würde sich beim Zurückdenken in die Zeit ohne elektronischen Literaturzugriff nicht mit Dankbarkeit an seine Bibliotheksaufenthalte erinnern? - ist über das klassische bibliothekarische Leistungsangebot nicht mehr aufrechtzuerhalten. Wer heutzutage elektronisch auf Leistungen seiner Bibliothek zurückgreift, ist sich häufig mangels persönlichen Kontakts kaum noch bewusst, welchen Vorarbeiten er die von ihm genutzten Leistungen $\mathrm{zu}$ verdanken hat. Die Begleitung der Forschenden im Forschungsdatenkreislauf ermöglicht es den Bibliotheken jedoch erneut, bei den Forschenden in zentralen Momenten ihrer Forschungstätigkeit unmittelbar präsent zu sein.

Die notwendigen Veränderungen bedingen ebenso eine kontinuierliche Neujustierung des überregionalen Zusammenspiels wissenschaftlicher Bibliotheken und des Zusammenwirkens von zentralen Fachbibliotheken und den dezentralen Bibliotheken. Was früher z. B. zentrale Sammlungsaufgabe war, ist heute zumeist besser dezentral darstellbar, zumal vielfach auch Gründe der Datensicherheit und des Datenschutzes gegen eine zu starke Zentralisierung von Datenbeständen sprechen. Umso mehr wachsen dafür zentrale Aufgaben bei der synergetischen Verknüpfung dezentraler Bestände. 


\section{Grundsätzlicher Paradigmenwechsel in der Informationserschließung und -verarbeitung steht bevor}

Darüber hinaus bedarf es aber auch eines grundsätzlichen Paradigmenwechsels bei der Erschließung und Vermittlung von Informationen. ${ }^{27}$ War es in Zeiten überschaubarer Mengen nutzbarer Information noch funktional, diese in ein oligodimensional-hierarchisch systematisierendes System einzuordnen, kann heute im Zeitalter der Datenflut und völlig neuer technischer Möglichkeiten ein solcher Ansatz nicht mehr hinreichen. Entsprechende Überlegungen wurden dabei keineswegs erst in allerjüngster Zeit angestellt. Bereits 1945 sprach Vannevar Bush in seinem berühmten Essay As We May Think ${ }^{28}$ von einem wachsenden Berg an Informationen, der in seiner Gänze für den Einzelnen immer weniger überschaubar wäre, sodass es neuer Methoden bedürfe, um Zusammenhänge erkennbar werden zu lassen. Diesen Gedanken entwickelte 1965 J. C. R. Licklider in Libraries of the Future ${ }^{29}$ weiter und hob hervor, dass es bereits mit der damals erreichten Fülle an Publikationen unmöglich geworden ist, tatsächlich auch alles zu lesen, ja, dass ein solcher Anspruch nicht einmal mehr ein effektives Vorgehen sei. Inzwischen hat die Situation drastisch an Dramatik gewonnen. ${ }^{30}$ Andererseits sind aber nicht nur die Möglichkeiten, solche Informationen $\mathrm{zu}$ gewinnen und abzuspeichern exponentiell gewachsen, sondern ebenso die technischen Möglichkeiten der Verarbeitung und Verknüpfung von Informationen. Ein besonders vielversprechender Zweig in der aktuellen Informatik sind die so genannten Wissensgraphen, mittels derer heterogene Wissensbestände in multiplen Dimensionen verknüpft und assoziativ Pfade zu relevanten, aber in klassischen Ordnungssystemen nicht erkennbar verknüpften anderweitigen Wissensbeständen eröffnet werden. Praktische Anwendungen dieses Grundansatzes finden sich bereits im Alltag, so werden bei einer Bestellung über Amazon Waren empfohlen, die völlig anderen Kategorien angehören, aber einen Nutzwert in Verbindung mit der eigentlich gesuchten Ware aufweisen. Ebenso führt das Social Network LinkedIn Nutzer sehr unterschiedlicher Herkunft zusammen, indem es Personen, bei denen gemeinsame Interes-

27 Vgl. den Überblick bei Decker, Stefan: Rethinking Access to Scientific Knowledge: Knowledge Graphs [veröffentlicht über LinkedIn], 16. August 2017. https://www.linkedin.com/pulse/ rethinking-scientific-knowledge-graphs-stefan-decker (21.12.2017).

28 Vgl. Bush, Vannevar: As We May Think. In: The Atlantic Monthly, Nr. 176, Juli 1945. S. 101-108.

29 Vgl. Licklider, J. C. R.: Libraries of the Future. Cambridge: MIT Press 1965.

30 Vgl. Bürger, Explosion (wie Anm. 1). 
sen vermutet werden, eine Kontaktaufnahme vorschlägt. Für die Verknüpfung von Forschungsdaten sind die Vorzüge dieses System besonders evident, da unter den heutigen Bedingungen erst unter Ausnutzung solcher technischen Möglichkeiten die ungeheure Menge aktuell verfügbaren Wissens auch praktisch erschlossen werden kann.

\section{Datenqualität bestimmt Wert als Rohstoff für die Forschung}

„FAIR Data“ als Goldstandard für Datenqualität: Die Nutzung der neuen Möglichkeiten setzt aber immer voraus, dass die Basis, auf die aufgebaut wird, tatsächlich trägt. Die Qualität von Daten hängt nicht nur von der Genauigkeit der Mess- und Aufzeichnungsmethoden sowie natürlich ihrer wissenschaftlichen Relevanz, sondern immer mehr auch von der Qualität ihrer Aufbereitung und Aufbewahrung ab. Dafür bürgert sich inzwischen der Begriff der „FAIR Data“ (nach dem englischen Akronym von Findable, Accessible, Interoperable, Reusable bzw. Reproducible) als allgemein akzeptierter generischer Standard ein. Dabei dürfen „FAIR Data“ nicht mit „Open Data“ verwechselt werden. „FAIR“ können Daten durchaus auch sein, wenn sie nicht frei öffentlich zugänglich sind, weil dem Datenschutzbelange, Rechte geistigen Eigentums, Geschäftsgeheimnisse oder das legitime Interesse des Datenerzeugers, mit seiner Auswertung der Daten als erster auf den wissenschaftlichen „Markt“ $\mathrm{zu}$ kommen und damit für die geleistete wissenschaftliche Arbeit die entsprechende Reputation zu erlangen, entgegenstehen. Auch beinhaltet „FAIR Data“ zwar eine Orientierung an guten wissenschaftlichen Praktiken, aber keineswegs eine Vereinheitlichung aller Datenschemata. Entscheidend ist vielmehr, dass die Daten so aufbereitet verfügbar sind, dass sie für berechtigte Nutzer möglichst niedrigschwellig auffindbar, zugänglich, verknüpfbar und weiterverwendbar sind. Es handelt sich also im Kern um einen Qualitätsanspruch an das Datenmanagement als Voraussetzung dafür, die oft mit hohem Aufwand gewonnenen Daten möglichst umfassend wissenschaftlich nutzen zu können. Der offene Zugang (,open access by default“) ist ein darüber hinausgehendes Desiderat offener Wissenschaft, das aber seinerseits nur effektiv wirken kann, wenn die Daten überhaupt erst einmal „FAIR“ aufbereitet vorliegen.

Organisatorische Gewährleistung von Datenqualität durch Datenintegrationszentren im Zuge der Nationalen Forschungsdateninfrastruktur flächendeckend ausbauen: Unerlässliche Voraussetzung für die praktische Gewährleistung von 
Datenqualität und „FAIR Data“ ist zudem deren institutionelle Absicherung durch auf diese Grundsätze verpflichtete organisatorische Strukturen. Solche Strukturen müssen sowohl nach außen wie nach innen wirkende Funktionen gewährleisten. Intern steht im Vordergrund, für eine geordnete und verlässliche Aufbewahrung der Daten zu sorgen, die qualitätsgerechte Aufbereitung der Daten nach den „FAIR“-Prinzipien sicherzustellen, mit hoher Kompetenz für Datenschutz und Datensicherheit Sorge zu tragen, die Wahrung von geistigen Eigentumsrechten und anderen schutzwürdigen wirtschaftlichen Belangen zu sichern, auf die Wahrung etwaiger Embargofristen der Forschenden zu achten und nicht zuletzt der jeweiligen Institution einen effektiven Einsatz der bei ihr gewonnenen Daten zu ermöglichen. Außerdem gilt es, die Forschenden von externen Anfragen zur Nutzung von Forschungsdaten zu entlasten. Gleichzeitig sind solche Datenintegrationszentren der One-Stop-Shop-Kontaktpunkt für Datenanfragen externer Forschender, der diesen einen, möglichst niedrigschwelligen Zugriff auf die Daten ermöglicht, sei es, dass die Daten an den Anfragenden exportiert werden, sei es, dass sie in anonymisierter, verschlüsselter oder aggregierter Form zur Verfügung gestellt werden oder sei es, dass lediglich nach den Vorgaben des Anfragenden erstellte Rechenergebnisse geliefert werden. Rollenmodell hierfür sind die vom Rat für Sozial- und Wirtschaftsdaten akkreditierten Forschungsdatenzentren. Ganz ähnlich werden derzeit im Zuge der Medizininformatik-Initiative des Bundesministeriums für Bildung und Forschung (BMBF) Datenintegrationszentren an den Hochschulkliniken etabliert. Die Bemühungen um eine Nationale Forschungsdateninfrastruktur bieten nunmehr die Chance, solche Forschungsdaten- oder Datenintegrationszentren flächendeckend in der Wissenschaftslandschaft zu etablieren.

Persistente Identifikatoren als Grundstein von Forschungsdatenmanagement: War früher nur ein Buch praktisch nutzbar, das im Katalog verzeichnet und im Regal systematisch richtig eingeordnet war, so sind heute Daten und Datensätze nur dann gesichert auffindbar, wenn sie mit persistenten Identifikatoren versehen sind. ${ }^{31}$ Die Verwendung persistenter Identifikatoren für Forschungsdaten ist deshalb bereits gegenwärtig als ein notwendiges Element guter wissenschaftlicher Praxis anzusehen.

Auch Forschungsdaten benötigen Gebrauchsanweisungen: Über die nackten Forschungsdaten hinaus bedarf es stets einer adäquaten Beschreibung mit Metada-

31 Vgl. Data Citation Synthesis Group: Joint Declaration of Data Citation Principles. Hrsg. von Maryann Martone. San Diego 2014. https://www.force11.org/group/joint-declaration-data-citation-principles-final (21.12.2017); Metadata Working Group: DataCite Metadata Schema for the Publication and Citation of Research Data. Version 3.1, Juni 2015. DOI: 10.5438/0010. 
ten, da nur so der Aussagehalt dieser Daten verstanden werden kann. Ähnlich wie bei archäologischen Fundstücken der jeweilige Grabungszusammenhang entscheidend für das Verständnis eines Fundstücks ist, müssen auch Forschungsdaten eingeordnet werden können, wenn sie sinnvoll verknüpft, aggregiert oder anderweit nachgenutzt werden sollen. Für dieses Anliegen haben sich Vorreiter wie Thomas Bürger z. B. in den Beiräten der europäischen Forschungsinfrastruktur DARIAH und der zentralen wissenschaftlichen Fachbibliothek TIB Hannover frühzeitig eingesetzt. Entscheidend für die Nutzbarkeit ist die Anreicherung der Forschungsdaten mit Metadaten und weiteren Annotierungen. Im Grunde handelt es sich dabei um die auf Forschungsdaten bezogene Fortschreibung traditioneller Bibliotheksarbeit der Nutzbarmachung in Form von Büchern und Aufsätzen publizierten Wissens. Allerdings wächst das entsprechende Aufgabenvolumen erheblich, da die auf Forschungsdaten bezogenen Ansprüche der Communitys vielfach sehr viel heterogener als bei klassischen Publikationen sind und das Anwachsen der Datenmengen den Aufwand für die Aufbereitung der Daten noch sehr viel stärker als das Publikationsaufkommen steigen lässt. Dies macht die Fortschreibung klassischer bibliothekarischer Aufgaben einschließlich der - wie im vorigen Abschnitt beschrieben - zu entwickelnden automatisierten Methoden nur umso dringlicher.

Standards nutzen, können aber auch schaden: Dabei ist die ambivalente Natur von Standardbildung zu betonen. So können Standards, die den Bedürfnissen einer Community hervorragend entsprechen, für die nächste Community mit zu grobem oder zu aufwendig feingliedrigem Raster oder mit unpassenden Kriterien versehen sein. Während für die eine Community größtmögliche Homogenität über die Zeit oder in der Fläche von Bedeutung ist, stellt für andere Communitys gerade die flexible Anpassung an sich zeitlich wandelnde oder an räumliche oder andere spezifische Gegebenheiten eine Notwendigkeit dar. Das Ziel kann deshalb nicht die größtmögliche Vereinheitlichung von Standards sein, da auf diese Weise nur für kaum jemanden noch brauchbare Minimallösungen entstehen würden.

Elastisches Gewebe von Thesauri, Ontologien und Sprachsystemen ist erforderlich: Notwendig erscheint vielmehr ein System, das für einen bestmöglichen Überblick über akzeptierte Standards und deren jeweiliges Anwendungsfeld sorgt, dynamische Weiterentwicklungen unterstützt und für eine effektive Verständigung der Subsysteme untereinander sorgt. Es verhält sich ganz ähnlich wie bei menschlichen Sprachen: Die Perspektive, dass weltweit nur noch eine Einheitssprache verwendet würde, wäre eine kulturelle Dystopie - in der Realität sind vielmehr Wörterbücher, Grammatiken, Beschreibungen kultureller Hintergründe, menschliche wie maschinelle Übersetzer effiziente und effektive Hel- 
fer zur Überwindung sprachlicher Barrieren, ohne den Reichtum einer Sprache ausmachende Besonderheiten abzuschleifen. Wenn sich unterschiedliche Kulturen von Disziplinen und Forschungsfeldern auch im Forschungsdatenmanagement eher noch weiter ausdifferenzieren als sich vereinheitlichen, werden hier ebenso Übersetzungsleistungen erforderlich, indem die in relevanten Communitys akzeptierten Standards systematisch erhoben, laufend aktualisiert und in Metadatenkatalogen oder vergleichbaren Übersichten erschlossen werden. Thesauri und Ontologien können gemeinsame Bezugspunkte schaffen, Konkordanzen und Diskordanzen, „Wörterbücher“ und „Grammatiken“ als strukturierte Übertragungshilfen die wechselseitige Verständigung über die Communitys hinweg erleichtern, automatisierte Annotierungen die Kommunikation als „Sprachassistenten“ unterstützen und Fachpersonal als „Dolmetscher“ den Austausch der Kommunizierenden mit Beratungsleistungen und Services unterstützen. $\mathrm{Zu}$ den europäischen Best-Practice-Beispielen in diesem Sinne gehört das aus dem BioMedBridge-Vorhaben hervorgegangene Corbel-Projekt großer lebenswissenschaftlicher Forschungsinfrastrukturen. ${ }^{32}$

Verantwortung von Bibliotheken: Die Empfehlungen der Hochschulrektorenkonferenz von 2015 machen besonders deutlich, dass genau hier eine besondere Verantwortung wissenschaftlicher Bibliotheken besteht. Wissenschaftliche Bibliotheken sind gefordert, ihr klassisches Know-how in diese neuen Aufgaben einzubringen und es kontinuierlich darauf bezogen weiterzuentwickeln. Nur so wird vermieden, dass sich ihre Anbindung an aktuelle Nutzerbedürfnisse lockert, nur so kann vor allem verhindert werden, dass Kompetenzen, die mit langer Tradition bereits in guter Qualität aufgebaut wurden, an anderer Stelle gedoppelt werden.

\section{Aufbau eines Systems für digitale Langzeit- archivierung steht noch am Anfang}

Digitale Langzeitarchivierung ist heutzutage ein weltweit ungelöstes Problem. Dies mag in Zeiten ständig wachsender und pro Speichereinheit immer günstiger werdender Speicherkapazitäten paradox klingen. Die zu bewältigenden Herausforderungen werden aber sofort deutlich, wenn man sich selber befragt, wie häufig man PC-Betriebssystem und Anwendungssoftware wechselt, ohne ältere Dokumente immer wieder auf den neuesten Stand zu bringen, inwieweit einem

32 www.corbel-project.eu (21.12.2017). 
bei der Migration auf einen neuen PC die vollständige Übertragung aller bisher gespeicherten Daten gelingt, inwieweit man noch Jahre später alle gespeicherten Daten wiederfindet, inwieweit man auch alle älteren Daten noch doppelt gesichert hält, inwieweit man seinen Datenbestand regelmäßig so aufräumt, dass ausschließlich die weiterhin relevanten Daten Speicherplatz beanspruchen ... Für Forschungsdaten stellen sich diese Herausforderungen noch verschärft, da ihr Volumen häufig viel höher ist, da sich der Bestand viel häufiger ändert, da die Heterogenität der Daten und der verwendeten Formate viel größer ist und nicht zuletzt, da zu ihrer Erstellung spezifische Software verwendet wurde, die später nicht nur schwer wiederzubeschaffen ist, sondern bei welcher ihrerseits die Kompatibilität mit den aktuell verwendeten Systemen zu gewährleisten ist, bei welcher ihre Weiterverwendbarkeit und Interpretierbarkeit von ihrer Kennzeichnung mit Metadaten entscheidend abhängt und bei welcher anders als beim privaten Datenschatz auch das Erinnerungsvermögen des Erzeugers der Daten zumeist nicht mehr herangezogen werden kann.

Alle diese Aufgaben stellen sich heute für Forschungsdaten an einer Vielzahl von Orten in ganz ähnlicher Weise, wobei die einzelne speichernde Institution bei der Entwicklung von Antworten und Lösungen vielfach auf wenig Vorlauf anderer Stellen zählen kann, sodass Parallelaufwand in hohem Maße stattfindet. Dabei gibt es im wissenschaftlichen Bibliothekswesen eine lange Tradition der institutionenübergreifenden Zusammenarbeit in der Bestandssicherung. So war die systematische Sammlung und Aufbewahrung relevanter Literatur lange Zeit in so genannten Sondersammelgebieten organisiert, die sich zwischenzeitlich im Zuge der Digitalisierung in Fachinformationsdienste gewandelt haben. Neben die Pflege und Aufbewahrung des Bestands an klassischen Publikationen tritt heute die Pflege und Aufbewahrung von Forschungsdaten. Dazu gehört die wichtige Funktion einer jeden Sammlungstätigkeit, die verfügbaren Ressourcen auf das besonders Erhaltenswerte zu konzentrieren und für weniger prioritäre Bestände sachgerechte Verfahren der Aussonderung zu etablieren. Insgesamt sieht auch die Empfehlung der Hochschulrektorenkonferenz bei der digitalen Langzeitarchivierung die wissenschaftlichen Bibliotheken speziell gefordert:

Besonders mit Blick auf [...] die Langzeitarchivierung[] können sich Hochschulen gegenüber anderen Anbietern als die geeigneten „Orte für Forschungsdaten“ positionieren. Im Vergleich zu projektförmigen Netzwerken [...] oder kommerziellen Anbietern [...] bieten die Repositorien der Hochschulen ein weitaus höheres Maß an Verlässlichkeit mit Blick auf den langfristig gesicherten Zugriff auf Datensätze. Speziell mit Blick auf die Dokumentation von Daten können die Bibliotheken der Hochschulen eine besondere, seit 
Langem bewährte Expertise der Informationsauszeichnung und -bewahrung in das FDM einbringen. ${ }^{33}$

Auch hier zählt die von Thomas Bürger geleitete SLUB zu den positiv hervorzuhebenden Best-Practice-Beispielen, ${ }^{34}$ so gehörte sie zu den gestaltenden Kräften in dem in Deutschland Maßstäbe setzenden nestor-Vorhaben. ${ }^{35}$ Perspektivisch wäre es eine attraktive Option, wenn sich als eine Konsequenz der derzeit laufenden Evaluierung des den Fachinformationsdiensten aktuell zugrunde liegenden Förderinstrumentariums ergäbe, dass diese gemeinsam mit den auf diesem Feld bereits tätigen drei zentralen Fachbibliotheken TIB, ZBW und ZB MED zusätzliche Aufgaben als Zentren für die digitale Langzeitarchivierung von Forschungsdaten übernehmen könnten.

\section{Der Schlüssel zur Hebung des Datenschatzes: Lehre, Informationskompetenz, Nachwuchsförderung, Datenwissenschaft}

Begriff der Informationskompetenz: Während im analogen Zeitalter Fragen der Informationskompetenz gut sortiert schienen, so herrscht heutzutage eine geradezu babylonische Verwirrung an Begrifflichkeiten und Konzepten. ${ }^{36}$ Dies

33 HRK, Empfehlung (wie Anm. 16), S. 12. Vgl. ferner Altenhöner, Reinhard: Digitale Langzeitarchivierung. In: Handbuch Bibliothek. Geschichte, Aufgaben, Perspektiven. Hrsg. von Konrad Umlauf u. Stefan Gradmann. Stuttgart: Metzler 2012. S. 173-186; Altenhöner, Reinhard u. Gert G. Wagner: Herausforderungen der Archivierung sozial-, verhaltens- und wirtschaftswissenschaftlicher Datenbestände. In: Langzeitarchivierung von Forschungsdaten. Standards und disziplinspezifische Lösungen. Hrsg. von Reinhard Altenhöner u. Claudia Oellers. Berlin: Scivero 2012. S. 15-18; Neuroth, Heike u. a. (Hrsg.): Langzeitarchivierung von Forschungsdaten. Eine Bestandsaufnahme. Boizenburg: Hülsbusch, Göttingen: Universitätsverlag 2012. http:// nbn-resolving.de/urn:nbn:de:0008-2012031401 (25.12.2017).

34 Vgl. z. B. Weckbrodt, Heiko: SLUB plant Langzeitarchiv für Sachsens Forschungsdaten. In: Dresdner Neueste Nachrichten vom 14. Juli 2016. http://www.dnn.de/Dresden/Lokales/SLUBplant-Langzeitarchiv-fuer-Sachsens-Forschungsdaten (25.12.2017).

35 Nestor: Digitale Langzeitarchivierung in einer Informationsinfrastruktur für Forschung und Bildung, 2015. http://files.dnb.de/nestor/berichte/nestor-Positionspapier.pdf (25.12.2017).

36 Vgl. Meyer-Doerpinghaus, Ulrich u. Heike Neuroth: Die Stärkung von Informationskompetenz im Kontext des Forschungsdatenmanagements: eine Herausforderung für Hochschulen und Politik. In: Zeitschrift für Bibliothekswesen und Bibliographie 62 (2015) H. 2. S. 80-84. DOI: 10.3196/186429501562237; Fühles-Ubach, Simone: Vom „embedded” zum „liaison librarian" - was versprechen die neuen Konzepte? In: Vernetztes Wissen - Daten, Menschen, Systeme. 6. Konferenz der Zentralbibliothek, Forschungszentrum Jülich 5.-7. November 2012. 
beginnt bereits damit, dass inzwischen schon der Begriff „Informationskompetenz“ selber vermehrt durch Begriffe wie „Digitalkompetenz“, „digitale Informationskompetenz“, „Datenkompetenz“ oder auch „Datenmanagementkompetenz“ ersetzt oder ergänzt wird. Dabei schließt der Begriff „Information“ jede Form verarbeiteter Daten ein, gleich ob digital oder analog, sodass diese neueren Begriffe letztlich jeweils Teilaspekte der für den Umgang mit Informationen heute notwendigen Kompetenzen hervorheben und damit Aufmerksamkeit für die sich wandelnden Inhalte und Gegenstände schaffen. Nur wer im digitalen Zeitalter auch mit digitalen Inhalten und insbesondere digitalen Forschungsdaten kompetent umzugehen vermag, besitzt heute die für eigenständiges wissenschaftliches Arbeiten erforderliche Informationskompetenz. Auch wenn also die Objekte, bezüglich welcher solche Kompetenz erforderlich ist, einem kontinuierlichen Wandel unterworfen sind und die damit verbundenen Herausforderungen immer weiter wachsen, so hat dies zwar sicherlich die Bedeutung von Informationskompetenz deutlich gesteigert, strukturell aber ist das Erfordernis, im gesamten Wissenskreislauf mit Informationen wissenschaftsadäquat umgehen $\mathrm{zu}$ können, weder neu noch objektgebunden. Am Begriff „Informationskompetenz" sollte deshalb festgehalten werden.

Sehr unterschiedliche Personengruppen und Aufgabenfelder sind zu adressieren: Relevanter für die Betrachtung des mit der Verbreitung und Entwicklung von Informationskompetenz verbundenen Aufgabenfelds ist aber, die sich derzeit stark ausdifferenzierenden und verändernden Anforderungen deutlich divergierender Personengruppen zu unterscheiden, nämlich (1) die Informatikfachleute mit spezifischer wissenschaftlicher Expertise für Speichern, Rechnen, Software, Artificial Intelligence, Deep Learning usw., (2) die Forschenden in allen anderen Disziplinen und Feldern, (3) die Datenwissenschaftler, die sich insbesondere den mit Forschungsdaten zusammenhängenden Infrastruktur und Dienstleistungsaufgaben widmen sowie (4) die allgemeine Öffentlichkeit. Zwar berühren und überschneiden sich die so gezogenen Kreise, doch erfordern sie eine jeweils spezifische Herangehensweise.

Hrsg. von Bernhard Mittermaier. Jülich: Forschungszentrum 2012, S. 337-350. http://www.dini. de/fileadmin/oa-statistik/projektergebnisse/Bibliothek_21.pdf (25.12.2017); Pampel, Heinz, Roland Bertelmann u. Hans-Christoph Hobohm: „Data Librarianship” - Rollen, Aufgaben, Kompetenzen. Working Paper Series des Rates für Sozial- und Wirtschaftswissenschaften, Nr. 144. Berlin 2010. http://www.econstor.eu/handle/10419/43622 (25.12.2017); Winkler-Nees, Stefan: Know-how der Bibliothekare gefragt. Die Virtualisierung der Wissenschaft stellt neue Aufgaben an die digitale Informationsversorgung. In: BuB - Forum Bibliothek und Information 63 (2011) H. 5, S. 373. 
Steigender Bedarf an IT-Fachkräften ist nur eines der zukunftsentscheidenden Themen: Dass der Bedarf an spezifischen IT-Fachkräften (1) weiter steigen wird, ist eine Mahnung, die nicht nur von der EU-Kommission immer wieder plakativ hervorgehoben wird. ${ }^{37}$ So unbestreitbar dieser Bedarf ist, so wenig darf er den Blick darauf verstellen, dass der Bedarf in den drei anderen genannten Kreisen von eher noch größerer Bedeutung ist. Bildlich gesprochen lässt sich das Forschungsdatenmanagement mit dem Autofahren vergleichen: Um das Mobilitätspotenzial des Autofahrens auszuschöpfen, bedarf es unzweifelhaft guter Ingenieure, die hochwertige, sichere und sparsame Fahrzeuge entwickeln. Noch wichtiger aber ist es, dass es eine Nutzerschaft gibt, die mit diesen Produkten nicht nur fahrtechnisch umgehen kann, sondern auch die damit verbundenen Risiken versteht und effektiv beherrscht, die für die Nutzung geltenden Regeln kennt und beachtet, Alternativen zum Autofahren sinnvoll abzuwägen versteht usw. Ähnliches gilt für das Forschungsdatenmanagement. Entscheidend sind auch hier nicht allein die eigenen technischen Infrastrukturfähigkeiten, sondern Fähigkeiten, die technisch eröffneten Möglichkeiten wirksam, effizient und verantwortungsbewusst einzusetzen. Hiermit sind die erwähnten Personengruppen (2) bis (4) angesprochen, die im Folgenden weiter abgehandelt werden sollen.

Informationen für die Öffentlichkeit als gesellschaftliche Aufgabe: Auf die spezifischen Aufgaben hinsichtlich der Information der allgemeinen Öffentlichkeit (4) kann an dieser Stelle allerdings in dem in diesem Beitrag gesetzten Rahmen lediglich sehr kurz eingegangen werden. Deshalb sei nur so viel gesagt, dass gerade die Flut begrenzt belastbarer oder gar irreführender Informationen auf Anbieterseite einer Begleitung durch vertrauenswürdige Orientierungen und Einordnungen bedarf sowie auf Nutzerseite Kritikfähigkeit und Kompetenz in der Nutzung von Orientierungs- und Einordnungsmöglichkeiten, wie sie Bibliotheken für klassische Literatur in langer Tradition vermitteln.

Informationskompetenz als unverzichtbare Querschnittskompetenz aller Forschenden: Ähnliches gilt für die Forschenden generell (2), für die wirksame, effiziente und verantwortungsbewusste Nutzung von Daten immer mehr zu einer in allen disziplinären Bereichen unabdingbar notwendigen Schlüsselkompetenz wird. Diese Kompetenzen müssen im Zuge des lebenslangen Lernens nicht nur in der Schule erworben und später in der Praxis den jeweiligen Not-

37 Vgl. bereits die Pressemitteilung von 2001: EU-Minister kommen zusammen, um das Problem der Qualifikationslücke anzugehen. http://europa.eu/rapid/press-release_IP-01-211_de. htm?locale=EN (21.12.2017). 
wendigkeiten angepasst werden, sondern darüber hinaus unabdingbarer Bestandteil jedes hochschulischen Curriculums werden. ${ }^{38}$

Informationskompetenz durch Datenwissenschaft - Datenwissenschaft als Querschnittsdisziplin: Etwas ausführlicher soll nunmehr auf die Datenwissenschaftler (3) eingegangen werden als demjenigen Fachpersonal, das sich speziell den mit Forschungsdaten zusammenhängenden Infrastruktur- und Dienstleistungsaufgaben sowie der methodischen und theoretischen Weiterentwicklung des Forschungsdatenmanagements widmet. Hierzu seien noch einmal die Empfehlungen Leistung aus Vielfalt des Rates für Informationsinfrastrukturen zitiert:

[Es werden] neue Formen der wissenschafts- und IT-nahen Datenkompetenz gebraucht. Fragen der Qualität, Produktivität und Integrität der Forschungsprozesse wie auch der Nachnutzbarkeit (oder ggf. Reproduktion) von Daten bedürfen arbeitsteilig-kooperativer Beurteilung. Für das Generieren von fachlichen und technischen Metadaten gilt Gleiches. Über Umfang und Art des Personalbedarfs für das umschriebene Feld - von der digitalen Bibliotheksexpertise über die Kuratierung von Forschungsdaten bis $\mathrm{zu}$ informatischen und fachmethodischen Schnittstellenkompetenzen - wird daher seit Jahren intensiv diskutiert. $^{39}$

Weiter heißt es:

Aufgrund des absehbar hohen Bedarfs sollten neue Studiengänge an einer großen Zahl von Hochschulen von den bestehenden bibliotheks-, archiv- bzw. informationswissenschaftlichen Zentren in enger Kooperation mit der Informatik und den jeweiligen Fachdisziplinen vor Ort entwickelt und angeboten werden. ${ }^{40}$

Zum Qualifikationsprofil dieser Berufsbilder hat die HRK in ihren Empfehlungen von 2012 ausgeführt, dass sie

sämtlich hohe Integrationsfähigkeiten [implizieren] und auf der Schnittstelle sowohl zwischen Wissenschaft und Infrastruktureinrichtungen als auch zwischen den Infrastruktureinrichtungen selbst [operieren], so dass die hergebrachte Trennung etwa zwischen Bibliothek und Rechenzentrum mit Blick auf jene Tätigkeit obsolet erscheint. ${ }^{41}$

Datenwissenschaftliche Einsatzfelder: Innerhalb der Gruppe der Datenwissenschaftler sind wieder diverse Einsatzfelder zu unterscheiden, nämlich (a) Auf-

38 Vgl. HRK, Empfehlung (wie Anm. 16), Ziff. 2.6.

39 RfII, Leistung aus Vielfalt (wie Anm. 18), S. 18.

40 RfII, Leistung aus Vielfalt (wie Anm. 18), S. 51.

41 Hochschulrektorenkonferenz: Entschließung der 13. Mitgliederversammlung der HRK am 20. November 2012 in Göttingen. Hochschule im digitalen Zeitalter: Informationskompetenz neu begreifen - Prozesse anders steuern. Bonn 2012, S. 16. http://www.hrk.de/uploads/media/Entschliessung_Informationskompetenz_20112012_01.pdf (25.12.2017). 
gaben, die unmittelbar in Forschungsvorhaben integriert, von Aufgaben, die von im Forschungsdatenmanagement Spezialisierten wahrgenommen werden, (b) Dateninfrastruktur-, Dienstleistungs- und Beratungsaufgaben auf Ebene von Fachbereichen, Fakultäten, Sonderforschungsbereichen, Exzellenz-Clustern und/oder der Hochschule insgesamt sowie (c) mit institutionell und organisatorisch übergreifenden Aufgaben Befasste.

Bei den zu (a) genannten Aufgaben setzt derzeit ein Spezialisierungsprozess ein, da das immer anspruchsvoller werdende Datenmanagement immer seltener nur mit für diesen Aufgabenbereich lediglich angelernten Kräften (etwa IT-affinen jungen Promovierenden, denen das Forschungsdatenmanagement als zusätzliche Nebenaufgabe zu ihrer „eigentlichen“ Forschungsarbeit übertragen wird) in guter Qualität zu gewährleisten ist. Zudem ist eine nachhaltige Datenpflege ausschließlich mit langfristig eingesetztem Infrastrukturpersonal sicherzustellen, da sich nur so fortlaufende Kompetenzabrisse bei jedem Wechsel zeitlich befristet beschäftigten wissenschaftlichen Personals vermeiden lassen.

Bei den zu (b) genannten Aufgaben setzt sich zunehmend die Erkenntnis durch, dass solche Aufgaben einer festen organisatorischen Verortung in Forschungsdatenzentren, Datenintegrationszentren oder anderen geordneten Strukturen bedürfen. Auf einige der damit verbundenen Anforderungen wurde bereits oben im Abschnitt Datenqualität eingegangen.

In das Einsatzfeld (c) gehören Infrastruktur-, Dienstleistungs- und Beratungsaufgaben sowie Aufgaben der Methodenforschung, wie sie insbesondere in den Abschnitten Dienstleistungen und digitale Langzeitarchivierung erörtert wurden und wie sie vor allem von zentralen Fachbibliotheken sowie FID-ähnlichen Strukturen zu organisieren sind.

Allen drei Feldern gemeinsam ist, dass dort forschungsdatenbezogene Spezialkenntnisse verlangt werden, die nicht nebenbei angelernt werden können, sondern von dafür besonders ausgebildeten und erfahrenen Fachkräften wahrgenommen werden müssen. Allerdings gibt es dafür bislang weder systematische Ausbildungs- oder Karrierewege noch ausformulierte Berufsbilder noch überhaupt etablierte Berufsbezeichnungen. Die Empfehlung der Hochschulrektorenkonferenz von 2015 besitzt deshalb unverminderte Aktualität:

Um zu einem guten institutionellen FDM zu kommen, ist es notwendig, die Kompetenzen aller Akteurinnen und Akteure an der Hochschule weiterzuentwickeln. Das gilt zunächst für die Lernenden, Lehrenden und Forschenden in allen Phasen ihres akademischen Lebens. Was die Informationskompetenz der Studierenden mit Blick auf FDM angeht, sollten entsprechende Module bzw. Modulelemente in sämtliche Studiengänge integriert werden. Die Informationsangebote der Bibliotheken und der Rechenzentren sollten noch mehr als bisher auf Fragen des FDM orientiert werden. [...] Damit die Mitarbeiterinnen und Mitarbeiter der Bibliotheken, Rechenzentren und E-Learning-Zentren sowie der 
Forschungs- und Rechtsreferate der Hochschulen in die Lage versetzt werden, die Wissenschaftlerinnen und Wissenschaftler beim FDM zu unterstützen, ist es notwendig, dass sie ihre Kompetenzprofile in Richtung des FDM erweitern. Von größter Bedeutung ist es, dass neue Studiengänge geschaffen werden, die auf Berufsprofile wie den Data Librarian oder den Data Scientist vorbereiten. ${ }^{42}$

Aufgaben wissenschaftlicher Bibliotheken: Dass die wissenschaftlichen Bibliotheken zur Erfüllung ihrer Aufgaben nicht nur auf eigene Informationskompetenz angewiesen sind, sondern ihrerseits zur Vermittlung von Informationskompetenz beitragen müssen, wird aus diesem Zitat bereits deutlich. Bedeutet dies nun, dass alle Bibliothekare künftig Informatiker werden müssen, wie ein Artikel $^{43}$ provokant fragt? Die Antwort hierauf muss - ebenso wie übrigens im erwähnten Artikel - differenziert ausfallen. Einerseits werden sich die in wissenschaftlichen Bibliotheken erforderlichen Qualifikationsprofile in der Tat deutlich verändern. In allen wissenschaftlichen Bibliotheken werden künftig neben der klassischen bibliothekarischen Kompetenz auch hinreichende Informatik- und disziplinäre Kompetenzen erforderlich sein. IT-Fähigkeiten sind dabei nicht nur notwendig, um digitale Entwicklungen im Alltag bewältigen zu können. Noch mehr werden sie gebraucht, um neue Möglichkeiten, wie sie durch technische Entwicklungen in immer rascherem Tempo entstehen, rechtzeitig zu erkennen und um sie strategisch für die Entwicklung des eigenen Angebotsportfolios nutzen zu können. Ebenso werden stärker noch als bisher fachliche, disziplinäre Kenntnisse benötigt, um ein attraktiver Begleiter der Wissenschaftlerinnen und Wissenschaftler bei ihren Forschungs-, Lehr- und Transferaufgaben sein zu können. Nicht nur sind Anforderungen und Kulturen in den verschiedenen Communitys sehr unterschiedlich, sondern vor allem auch hier in einem immer rascheren Wandel begriffen. Zum Dritten bleibt es aber ebenso unabdingbar, dass die besondere bibliothekarische Ordnungs-, Systematisierungs- und Orientierungskompetenz weiterhin in guter Qualität zum Tragen gebracht wird, da - wie bereits in den vorausgehenden Abschnitten wiederholt illustriert - nur mit diesen Fähigkeiten potenziell gegebene Möglichkeiten effektiv und zielgerichtet genutzt werden können.

42 HRK, Empfehlung (wie Anm. 16), S. 14-15.

43 Becker, Pascal-Nicolas u. Fabian Fürste: Sollen wir Bibliothekare jetzt alle Informatiker werden? Forschungsdatenmanagement, Datenerhaltung und -pflege als neue Aufgabenfelder. In: BuB - Forum Bibliothek und Information 65 (2013) H. 7-8. S. 512-514. http://b-u-b.de/sollen-wir-bibliothekare-jetzt-alle-informatiker-werden/ (25.12.2017). 


\section{Ausblick: Transformation als Dauer- und Normalzustand}

Ein weiteres, häufig unterschätztes Charakteristikum des gegenwärtigen Umbruchs ist, dass es sich beim digitalen Wandel nicht etwa um einen einmaligen Umstieg von gedruckter auf digitale Literatur usw. handelt, sondern um einen dynamischen Prozess, dessen Ende nicht nur nicht absehbar ist, sondern der sich immer weiter beschleunigt, in dem immer kürzere Innovationszyklen zu bewältigen sind, in dem immer neue Möglichkeiten genutzt werden können, in dem allein schon die Bewahrung des bereits erzeugten Wissens zu einer immer schwieriger zu bewältigenden Herausforderung wird. In dieser Situation sind Bibliotheken nicht primär gefragt, die Erfüllung ihrer traditionellen Aufgaben auf digitale Wege umzustellen. Im Vordergrund steht vielmehr, dass Bibliotheken ihre Fähigkeiten einbringen, zu systematisieren und $\mathrm{zu}$ ordnen und so Orientierung in der Unendlichkeit und stetigen Wechselhaftigkeit des Datenmeers vermitteln sowie dass Bibliotheken ihre Rolle als gute Begleiter ihrer Nutzerinnen und Nutzer bei der Bewältigung der Dynamik aktiv annehmen und ihre Nutzerschaft sowohl bei der Lösung der damit verbundenen Herausforderungen als auch bei der Nutzung der darin liegenden Chancen kompetent unterstützen. Genau wie ihrer Nutzerschaft steht damit den wissenschaftlichen Bibliotheken eine lang anhaltende Phase ununterbrochener Transformationen bevor. Agilität und frühzeitiges Erkennen und Aufgreifen neuer Herausforderungen werden so zu Schlüsselkompetenzen. Wissenschaft ist überdies von Natur aus global; die neuen technischen Möglichkeiten fördern das grenzüberschreitende Zusammenwachsen von Wissenschaft noch weiter. Dementsprechend müssen wissenschaftliche Bibliotheken ihre Nutzerschaft auch insoweit auf ihrem Weg begleiten sowie ihre Entwicklung von Beständen, Dienstleistungen und weiteren Angeboten stets auch aus einer europäischen und internationalen Perspektive betrachten.

Die Bibliotheken tragen für die Bewältigung der Aufgaben rund um das Forschungsdatenmanagement eine herausgehobene Verantwortung. Sie sind nicht nur - wie im Verlauf dieses Beitrags mit zahlreichen Zitaten belegt - mit erheblichen Erwartungshaltungen aus der Wissenschaft konfrontiert, sondern verfügen zur Wahrnehmung dieser Verantwortung über besondere Kernkompetenzen, Erfahrungen und Strukturen, die für eine erfolgreiche Gestaltung des Forschungsdatenmanagements unabdingbar sind. Sie tragen diese Verantwortung nicht allein, sondern sind immer auf eine enge Kooperation insbesondere mit den Rechenzentren der Hochschulen sowie den verschiedenen disziplinären Wissenschaften angewiesen. 